\title{
Differentiation of cerebrospinal fluid inflammatory biomarkers between neonatal post-hemorrhagic and congenital hydrocephalus
}

\author{
Gakwaya Habiyaremye ${ }^{1 *}$, Diego M Morales ${ }^{1}$, Clint D Morgan², James P McAllister ${ }^{1}$, David D Limbrick \\ From Hydrocephalus 2015 \\ Banff, Canada. 18-21 September 2015
}

\section{Introduction}

Neonatal Post-hemorrhagic hydrocephalus (PHH) develops partly due to an inflammatory process occurring after intraventricular hemorrhage whereas the majority of congenital hydrocephalus $(\mathrm{CH})$ results from primary and secondary CNS malformations. We hypothesize that cerebrospinal fluid (CSF) content of inflammatory biomarkers is higher in neonatal $\mathrm{PHH}$ relative to $\mathrm{CH}$. To test this hypothesis, we measured CSF concentrations of CCL-3, CXCL-12, CX3CL-1, IL-10 and P-selectin in both conditions.

\section{Methods}

ELISA was used to measure CSF inflammatory biomarker concentrations in 10-15 patients per study group. Study groups included PHH-LP (lumbar puncture), congenital hydrocephalus $(\mathrm{CH})$, and pre-term controls (PT). PHH-LP and PT samples were collected perinatally during spinal tap; $\mathrm{CH}$ samples were collected ventricularly at time of reservoir implantation.

\section{Results}

CCL-3 was significantly increased in $\mathrm{PHH}$ relative to both $\mathrm{CH}$ and PT (PHH-LP>CH with $\mathrm{p}=0.0002$; $\mathrm{PHH}-$ $\mathrm{LP}>\mathrm{PT}, \mathrm{p}=0.0001)$. $\mathrm{P}$-selectin was significantly elevated in $\mathrm{PHH}$ compared to $\mathrm{CH}$ and $\mathrm{PT}$ ( $\mathrm{PHH}-\mathrm{LP}>\mathrm{CH}$, $\mathrm{p}=0.0002 ; \mathrm{PHH}-\mathrm{LP}>\mathrm{PT}, \mathrm{p}=0.0009)$. IL-10 was significantly elevated in PHH-LP relative to PT (PHH-LP $>$ PT, $\mathrm{p}=0.0001$ ). No significant differences were found in CXCL-12 and CX3CL-1. Study group comparison did not show any difference between $\mathrm{CH}$ and PT.

\section{Conclusions}

Our findings suggest that PHH may be distinguished from $\mathrm{CH}$ based on its higher levels of CCL-3 and P-selectin. However, CX3CL-1, CXCL-12 and IL-10 may not be useful in distinguishing both conditions. Interestingly, it appears that $\mathrm{CH}$ may not be distinguished from PT controls based on levels of inflammatory biomarkers. These findings confirm our hypothesis that inflammatory biomarkers are higher in neonatal $\mathrm{PHH}$ and indicate that the use of inflammatory modulators could be more beneficial to $\mathrm{PPH}$ versus $\mathrm{CH}$.

\section{Authors' details}

'Department of Neurological Surgery, Division of Pediatric Neurosurgery, Washington University, St. Louis, MO, USA. ${ }^{2}$ Vanderbilt University School of Medicine, Nashville, TN, USA.

Published: 18 September 2015

doi:10.1186/2045-8118-12-S1-P15

Cite this article as: Habiyaremye et al:: Differentiation of cerebrospinal fluid inflammatory biomarkers between neonatal post-hemorrhagic and congenital hydrocephalus. Fluids and Barriers of the CNS 2015 12(Suppl 1): P15.

\footnotetext{
* Correspondence: habiyaremyeg@wudosis.wustl.edu

'Department of Neurological Surgery, Division of Pediatric Neurosurgery,

Washington University, St. Louis, MO, USA

Full list of author information is available at the end of the article
} 